\title{
A data-based approach for multivariate model predictive control performance monitoring is
}

\author{
Xuemin Tian ${ }^{\mathrm{a}}$, Gongquan $\mathrm{Chen}^{\mathrm{a}}$, Sheng Chen ${ }^{\mathrm{b}, *}$ \\ a College of Information and Control Engineering, China University of Petroleum (Hua Dong), Donying, Shandong 257061, China \\ ${ }^{\mathrm{b}}$ School of Electronics and Computer Science, University of Southampton, Highfield, Southampton SO17 1BJ, UK
}

\section{A R T I C L E I N F O}

\section{Article history:}

Received 18 February 2010

Received in revised form

6 September 2010

Accepted 17 September 2010

Communicated by J. Zhang

Available online 20 October 2010

Keywords:

Model predictive control

Performance monitoring

Performance assessment

Performance diagnosis

Eigenvector angle based classifier

Intelligent system

\begin{abstract}
A B S T R A C T
An intelligent statistical approach is proposed for monitoring the performance of multivariate model predictive control (MPC) controller, which systematically integrates both the assessment and diagnosis procedures. Model predictive error is included into the monitored variable set and a 2-norm based covariance benchmark is presented. By comparing the data of a monitored operational period with the "golden" user-predefined one, this method can properly evaluate the performance of an MPC controller at the monitored operational stage. Characteristic direction information is mined from the operating data and the corresponding classes are built. The eigenvector angle is defined to describe the similarity between the current data set and the established classes, and an angle-based classifier is introduced to identify the root cause of MPC performance degradation when a poor performance is detected. The effectiveness of the proposed methodology is demonstrated in a case study of the Wood-Berry distillation column system.
\end{abstract}

(c) 2010 Elsevier B.V. All rights reserved.

\section{Introduction}

Most controllers operate well at the very early stage of plant operation because of initial controller tuning at the beginning of production but their performance deteriorate after a period of production time owing to a variety of causes, including the variation of raw material property, plant process model mismatch, fluctuation of disturbances, malfunction of sensor and actuator, and improper maintenance. Therefore, controller performance monitoring is essential to ensure high performance of industrial control systems. Since the initial work by Harris [1] on the minimum variance control (MVC) benchmark, many research interests have been focused on the performance assessment of single-input single-output (SISO) and multiple-input multipleoutput (MIMO) controllers as well as feedback, forward and cascade controllers, resulting in a large amount of publications [2-7] and several commercial applications [8,9]. Development of modern industry, such as oil-refinery, petrol-chemistry, metallurgy and pharmacy, has stimulated the implementation of advanced process control applications, including the model predictive control (MPC) [10]. The MPC strategy has the ability to run an industrial

\footnotetext{
This work is supported by the National 863 Program of China (no. 2007AA04Z193).

* Corresponding author.

E-mail addresses: tianxm@hdpu.edu.cn (X. Tian), sqc@ecs.soton.ac.uk(S. Chen).
}

process close to its limits [10] and is currently regarded as one of the most popular industrial control strategies.

Increasing popularity of MPC in industrial applications has led to high demand for performance assessment and diagnosis of MPC systems. The task of MPC controller performance monitoring is composed of three steps. Firstly, the capability of the MPC control system is determined by defining an appropriate benchmark to which the MPC control system will be compared. Secondly, proper monitoring statistics are constructed to assess the controller, in order to make sure whether the MPC controller is operating in a right status and whether its key performance index satisfies the designed performance. Thirdly, methods are established for diagnosing the root causes of performance degradation when poor performance of MPC controller is detected. The existing works on performance monitoring of MPC controllers, however, mainly focus on the first two steps of performance monitoring, namely, performance assessment, and not enough attention has been paid to the third step of performance diagnosis. Moreover, in a few existing reports on industrial applications of MPC performance assessment, such as [8,9], the widely adopted method is the MVC benchmark. However, the MVC benchmark used for the performance monitoring of feedback controllers is not well suited to the performance monitoring of MPC systems, as the constraints and nonlinearity of the MPC algorithm make the theoretical MVC lower bound unrealisable [11-21].

Since the MVC benchmark is impractical, some alternatives have been adopted to evaluate MPC controllers. The multivariate impulse response plot [11] provides a graphical measure in terms 
of settling time, decay rate and other deterministic controller performance indicators, and this method is applied to evaluate MPC controllers in [9]. Huang [12] and recently Zhao et al. [13] propose to use the linear quadratic Gaussian (LQG) control as the benchmark, while Patwardhan and Shah [14] apply this method for MPC performance assessment, at the cost of solving the complicated LQG problem. Multivariate statistic methods, such as principal component analysis (PCA) and partial least squares, are introduced to controller performance assessment in $[15,16]$. The authors of [17] propose a performance index based on the comparison of the designed and achieved objective functions. Similarly, the authors of [18] propose two performance indexes, with the ratio of historical and achieved performance for monitoring while the ratio of designed and achieved performance for diagnosis. Diagnosis presented in [18], however, is very limited and can only indicate whether the problem is caused by the manipulated variable or not. In $[19,20]$, techniques are developed using the data-based covariance benchmark for performance assessment. In particular, a performance index is derived as the product of generalised eigenvalues in [20], while the method for performance diagnosis presented in the accompanied work [21] is based on the variable separation approach from the process fault diagnosis.

Methods also exist to evaluate the MPC controller directly without using a performance index. In [22,23], neural network and PCA similarity based classifiers are utilised, respectively, to classify the MPC performance into four classes in a diagnosis procedure. The authors of [14] quantify the effect of constraints, modelling uncertainty, disturbance uncertainty and process nonlinearity on the closed loop performance. The disadvantage of the above methods is that the performance status cannot be estimated quantitatively. The authors of [24] compare the performance of a MPC controller with an ideal controller which inherits the structure, constraints and tuning parameters of the controller been evaluated. But it is complicated to build such an ideal controller. The work [25] discusses the causes of plant-wide disturbances which may influence the performance of controllers, while the authors of [26] use the correlation between a dithering signal and the prediction error for detecting model mismatch in the process of monitoring. The authors of [27] take the predictive error into consideration in performance assessment. The works $[15,28]$ use the likelihood method to locate the cause of performance degradation. The work [29] presents an SISO MPC performance curve to determine whether the poor performance is caused by the controller itself or by the plant model. Huang [30] establishes a Bayesian framework to analyse whether each of the four control-loop components works at a satisfied status. This approach does not consider performance assessment.

With the development of modern measurement and distributed control system (DCS) techniques, abundant plant process data can be easily acquired and stored, which include process valuable information that have not been sufficiently used. Hence, many user-defined data-based statistical methods are proposed and user-defined covariance benchmarks are preferred in performance assessment. It can also be observed from the existing works that many techniques proposed lack effective performance diagnosis component or assessment and diagnosis components are not coherently integrated. Against this background, in this paper we propose a coherent statistical approach for MPC performance monitoring, which integrates all the stages of performance monitoring procedure. Specifically, a user-defined 2-norm based covariance benchmark is presented to evaluate the performance of MPC controller. When performance degradation is detected, the performance diagnosis procedure is carried out to locate the causes using pattern classification according to the user-defined principal angle based method. A case study of the Wood-Berry distillation column system is demonstrated using the proposed performance monitoring framework. While our method is clearly motivated by some previous works and shares some common connections with them, important differences exist between our approach and the previous works.

We now emphasise our novel contributions, in comparison with the previous key works. The diagnosis method presented in [18] only distinguishes the two classes of performance deterioration, namely, those problems associated with the controller and those problems not caused by the controller. Any further diagnosis of root causes can only be done by manual inspection and carrying out further model validation. In contrast to this complicated procedure by human operator, our monitoring procedure can automatically locate the root cause of performance deterioration. Yu and Qin [20,21] developed an important data-driven framework for performance assessment and diagnosis. While the determinant-based covariance benchmark derived in [20] proves to be very effective in performance assessment, it requires that the covariance matrix of the monitored data must be nonsingular. By contrast, our 2-norm based covariance benchmark has no such limitation. Performance diagnosis of MPC systems proposed in [21] is based on the method of loading-based and angle-based contributions, which focuses on the variable identification. Specifically, the method of [21] identifies those variables which contribute significantly to the inflated or reduced variance. The exact root cause, however, cannot be given since there are potentially many causes for the inflated or reduced variance. Thus, this method of contribution plot provides engineer less information regarding the poor performance of control loops than our proposed diagnosis method, which directly identifies the root cause of performance deterioration. The diagnosis procedure of $[22,23]$ employs three binary classifiers to classify the MPC performance into four classes. Moreover, the method of [22,23] does not include a quantitative performance index for assessing the level of performance deterioration.

The reminder of this contribution is organised as follows. After a brief review of the existing determinant-based covariance benchmark, Section 2 proposes a data-driven 2-norm based covariance benchmark for systematically carrying out performance assessment. A performance diagnosis technique using the user-defined principal angle based classification method is presented subsequently in Section 3. Section 4 provides a case study to illustrate the effectiveness of the proposed performance monitoring framework, and our conclusions are offered in Section 5.

\section{Performance assessment using data-driven covariance benchmark}

In developing a data-driven covariance benchmark for MPC performance assessment $[7,20]$, a period of reference data is chosen as a user-specified benchmark, which should be a period of "golden" operation data from the process with satisfactory control performance [7]. Let the benchmark period be denoted by I and the monitored period by II. Further denote the reference data as $\mathbf{y}_{\mathrm{I}}$ and the data of the monitored period by $\mathbf{y}_{\mathrm{II}}$. With the definition of the covariance matrix $\operatorname{cov}(\mathbf{y})=E\left\{\mathbf{y} \mathbf{y}^{T}\right\}$, where $E\{\bullet\}$ is the expectation operator, and assuming that $\operatorname{cov}\left(\mathbf{y}_{\mathrm{I}}\right)$ and $\operatorname{cov}\left(\mathbf{y}_{\mathrm{II}}\right)$ have the full rank $q$, the performance index of $[7,20]$ is defined by

$\eta_{\mathrm{det}}=\frac{\left|\operatorname{cov}\left(\mathbf{y}_{\mathrm{I}}\right)\right|}{\left|\operatorname{cov}\left(\mathbf{y}_{\mathrm{II}}\right)\right|}=\prod_{i=1}^{q} \lambda_{i}=|\boldsymbol{\Lambda}|$,

where $|\bullet|$ denotes the matrix determinant operator, $\lambda_{i}$ are the $i$ th generalised eigenvalue between $\operatorname{cov}\left(\mathbf{y}_{\mathrm{I}}\right)$ and $\operatorname{cov}\left(\mathbf{y}_{\mathrm{II}}\right)$, and $\boldsymbol{\Lambda}=\operatorname{diag}\left\{\lambda_{1}, \lambda_{2}, \ldots, \lambda_{q}\right\}$ is the diagonal matrix consisting of the generalised eigenvalues. The performance assessment using $\eta_{\text {det }}$ can be summarised as follows [20].

(1) If $\eta_{\text {det }}$ is smaller than 1 , the performance of the monitored period II is considered to have deteriorated significantly from the expected benchmark period I. 
(2) If $\eta_{\text {det }}$ is close to 1 , the performance of the monitored period II is believed to be unchanged or as good as that of the benchmark period I.

(3) If $\eta_{\text {det }}$ is larger than 1, the performance of the monitored period II is better than the benchmark. Then the monitored period II could be chosen as the new benchmark.

The determinant-based covariance benchmark (1) is an important practical benchmark that motivates our alternative userdefined benchmark. A problem associated with the data-driven performance assessment (1) is that the eigendirections or subspaces identified by the data-driven covariance benchmark (1) are not very useful for performance diagnosis and could not be used to identify the exact root cause of the poor performance detected [21]. The requirement of a full-rank covariance matrix may also cause some practical difficulty.

As a model-based control strategy, MPC computes the future control actions by minimising an objective function over a finite prediction horizon according to the historical information and future input of the process model. Usually, the quadratic objective function is selected as

$J(k)=E\left\{\left(\mathbf{y}(k+1)-\mathbf{y}_{\mathrm{r}}(k+1)\right)^{T} \mathbf{Q}\left(\mathbf{y}(k+1)-\mathbf{y}_{\mathrm{r}}(k+1)\right)+\Delta \mathbf{u}^{T}(k) \mathbf{R} \Delta \mathbf{u}(k)\right\}$,

where $\mathbf{y}(k)$ and $\mathbf{y}_{\mathrm{r}}(k)$ denote the output variables and reference trajectory at the $k$ step, respectively, $\Delta \mathbf{u}(k)$ represents the increment of the manipulated variables, $\mathbf{Q}$ is a positive definite weighting matrix and $\mathbf{R}$ a nonnegative definite one. From (2), it can be seen that the first part of $J(k)$ is related to the covariance matrix of the output variables $\mathbf{y}(k)=\left[y_{1}(k) y_{2}(k) \cdots y_{n}(k)\right]^{T}$, while the second part is associated with the covariance matrix of the input variables $\mathbf{u}(k)=\left[u_{1}(k) u_{2}(k) \cdots u_{m}(k)\right]^{T}$. Therefore, both $\mathbf{y}(k)$ and $\mathbf{u}(k)$ should be included in the monitored variable set. Moreover, the manipulated variable increments can be expressed as

$\Delta \mathbf{u}(k)=\left(\mathbf{A}^{T} \mathbf{Q A}+\mathbf{R}\right)^{-1} \mathbf{A}^{T} \mathbf{Q}\left(\mathbf{y}_{\mathrm{r}}(k+1)-\mathbf{A}_{0} \mathbf{u}(k-1)-\mathbf{H e p}(k)\right)$,

where $\mathbf{A}$ and $\mathbf{A}_{0}$ are the matrices determined by the process model, $\mathbf{H}$ is the feedback correction factor matrix, and $\mathbf{e p}(k)=$ $\left[e p_{1}(k) e p_{2}(k) \cdots e p_{n}(k)\right]^{T}$ is the model predictive error vector. Referring to the internal model control based MPC controller depicted in Fig. 1, when the set-point of the controlled variable is set to zero, the model predictive error can be expressed as

$\mathbf{e p}(z)=\mathbf{y}(z)-\hat{\mathbf{y}}(z)=\mathbf{G}_{\mathrm{v}}(z) \mathbf{v}(z)-\left(\mathbf{G}_{\mathrm{p}}(z)-\mathbf{G}_{\mathrm{m}}(z)\right) \mathbf{G}_{\mathrm{c}}(z) \mathbf{e p}(z)$,

where $z$ denotes the $Z$-transform variable, $\mathbf{G}_{\mathrm{p}}(z), \mathbf{G}_{\mathrm{v}}(z), \mathbf{G}_{\mathrm{m}}(z)$ and $\mathbf{G}_{\mathrm{c}}(z)$ are the plant, disturbance, model and controller transfer function matrices, respectively. Thus, we obtain

$\mathbf{e p}(z)=\left(\mathbf{I}+\left(\mathbf{G}_{\mathrm{p}}(z)-\mathbf{G}_{\mathrm{m}}(z)\right) \mathbf{G}_{\mathrm{c}}(z)\right)^{-1} \mathbf{G}_{\mathrm{v}}(z) \mathbf{v}(z)$,

where I denotes the identity matrix. It can be seen from (5) that the model predictive error is affected not only by the control action but also the level of process-model mismatch as well as the plant disturbances. In principle, therefore, any MPC controller

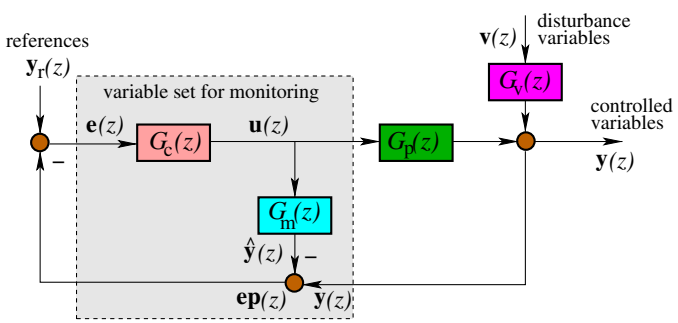

Fig. 1. Schematic diagram of the internal model control structure for model predictive control. performance deterioration as the result of process and/or disturbance changes can be detected and identified by the model predictive errors. Thus, the model predictive errors together with the controlled variables and manipulated variables should be included in the monitoring variable set, and we establish the extended monitored variable set consisting of the following variables:

$\tilde{\mathbf{y}}=\left[u_{1} \cdots u_{m} e p_{1} \cdots e p_{n} y_{1} \cdots y_{n}\right]^{T}$.

We adopt a user-defined data-based approach. Again denote the benchmark period by I and the monitored period by II. The basic idea of our method comes from the PCA, which is a dimensiondeduction method used to look for the main data characteristics. Through singular value decomposition (SVD) of the covariance matrix of the monitored data, the principal eigenvector corresponding to the maximum eigenvalue reflects the most significant performance direction information, and the corresponding maximum eigenvalue provides the maximum explanation of the performance variation. Specifically, our proposed performance index is defined by

$\eta_{\mathrm{m} 2 \mathrm{n}}=\frac{\left\|\operatorname{cov}\left(\tilde{\mathbf{y}}_{1}\right)\right\|_{2}}{\left\|\operatorname{cov}\left(\tilde{\mathbf{y}}_{\mathrm{II}}\right)\right\|_{2}}$

where $\|\bullet\|_{2}$ denotes the matrix 2-norm, $\operatorname{cov}\left(\tilde{\mathbf{y}}_{\mathrm{I}}\right)$ and $\operatorname{cov}\left(\tilde{\mathbf{y}}_{\mathrm{II}}\right)$ represent the covariance matrices of the selected benchmark period and the monitored period, respectively. Unlike the performance index (1), we do not required that $\operatorname{cov}\left(\tilde{\mathbf{y}}_{\mathrm{I}}\right)$ and $\operatorname{cov}\left(\tilde{\mathbf{y}}_{\mathrm{II}}\right)$ have a full rank. Denote the covariance matrix of $\tilde{\mathbf{y}}$ as $\mathbf{C}=\operatorname{cov}(\tilde{\mathbf{y}})$. Obviously, $\|\mathbf{C}\|_{2}$ is just the square root of the maximum eigenvalue of $\mathbf{C}^{T} \mathbf{C}$. Let a SVD of $\mathbf{C}$ be expressed by

$\mathbf{C}=\boldsymbol{\Phi}\left[\begin{array}{ll}\mathbf{D} & \mathbf{0} \\ \mathbf{0} & \mathbf{0}\end{array}\right] \boldsymbol{\Phi}^{T}$,

where $\quad \mathbf{D}=\operatorname{diag}\left\{\sigma_{1}(\mathbf{C}), \sigma_{2}(\mathbf{C}), \ldots, \sigma_{r}(\mathbf{C})\right\}, \quad \sigma_{1}(\mathbf{C}) \geq \sigma_{2}(\mathbf{C}) \geq \cdots \geq$ $\sigma_{r}(\mathbf{C})>0$ are the nonzero singular values of $\mathbf{C}$, and $\boldsymbol{\Phi}$ is an orthonormal matrix consisting of the associated singular vectors. As the singular values are arranged in descending order, the maximum singular value is simply $\sigma_{\max }(\mathbf{C})=\sigma_{1}(\mathbf{C})$. Thus, the 2-norm performance index (7) can be expressed as

$\eta_{\mathrm{m} 2 \mathrm{n}}=\frac{\sigma_{\max }\left(\mathbf{C}_{\mathrm{I}}\right)}{\sigma_{\max }\left(\mathbf{C}_{\mathrm{II}}\right)}$

where $\mathbf{C}_{\mathrm{I}}=\operatorname{cov}\left(\tilde{\mathbf{y}}_{\mathrm{I}}\right)$ and $\mathbf{C}_{\mathrm{II}}=\operatorname{cov}\left(\tilde{\mathbf{y}}_{\mathrm{II}}\right)$. Although the benchmark $\eta_{\mathrm{det}}$ of (1) generally performs slightly better (more sensitive) than $\eta_{\mathrm{m} 2 \mathrm{n}}$ in the performance assessment of MPC systems, our performance assessment method offers advantage for performance diagnosis. The eigenvector associated with the maximum eigenvalue of $\mathbf{C}_{\mathrm{II}}^{T} \mathbf{C}_{\mathrm{II}}$, obtained in the performance assessment stage is utilised directly in the performance diagnosis stage to locate the root cause of the performance deterioration detected. This will be explained later in the next section.

The proposed performance assessment approach is shown within the framework of data-based MPC controller performance monitoring depicted in Fig. 2. From abundant process data contained in the DCS database, appropriate benchmark data are extracted based on the experience or prior-knowledge on the process control system, and the corresponding benchmark is established. Then the performance index is calculated to evaluate the current control system been monitored. The performance assessment procedure is summarised as follows:

(1) If the performance index is smaller than 1, the performance of the monitored MPC controller is considered to have deteriorated significantly from the expected benchmark performance. Then the diagnosis procedure is entered. 
(2) If the index is close to 1 , the current performance is believed to be unchanged or as good as that of the benchmark, and the repeated operation is carried out to evaluate the performance of the next period.

(3) If the index is observed to be larger than 1 , the current performance of the MPC controller is better than that of the benchmark selected before. Therefore, the benchmark should be updated using the current data.

\section{Performance diagnosis based on classifier}

We first analyse the factors which influence the performance of MPC control systems and divide them into several classes. This requires to collect sample data for each class according to the priorknowledge and experience of the controlled system, and to perform a SVD on the data to extract the class representative feature in terms of the principal eigenvector of the data covariance matrix. When the performance index shows that the controller does not work at the expected status, the MPC controller performance diagnosis, as shown in the bottom part of Fig. 2, is called to determine the cause for the performance degradation detected. This involves recognising the class of the current controller performance by means of the eigenvector angle based classifier.

\subsection{Classification of performance deterioration causes}

Generally, causes that can lead to the performance degradation of MPC controllers include property variation of raw material, plant process-model mismatch, fluctuation of disturbances, malfunction of actuators and sensors, and improper maintenance. In the tree of performance deterioration causes illustrated in Fig. 3, we classify the causes of MPC performance deterioration into the two groups

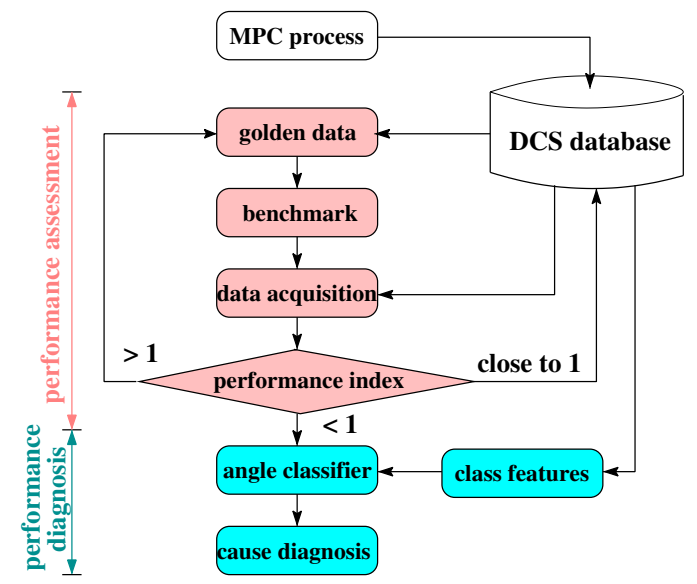

Fig. 2. Data-based MPC controller performance monitoring framework. due to internal and external factors, respectively. Within the group of internal factors, further divisions are made. The plant process model may change because of the changes in raw material properties, rust of equipment, influence of seasons, wear and tear of pipe, activity of catalyst and so on. This may lead to the model mismatch reflected in the static gain and time constant parameters. Changes in the controller constraints and poor controller tuning can also be considered to be the internal causes. Similarly, external factors include the variety of the measured and unmeasured disturbances due to stochastic influence which cannot be predicted in advance. Furthermore, there are some other external causes which also lead to poor performance, such as the valve stiction, malfunction of actuators and sensors. Proper classification of various causes is an important research subject entirely in itself.

In order to locate the root cause of performance deterioration, information reflecting each cause should be extracted. A data-based method for performance monitoring should pay particular attention to yielding meaningful performance directions from the process data [20]. Assume that the $g$ causes or classes of performance deterioration are established, which are marked as $C_{1}, C_{2}, \ldots, C_{g}$, according to the prior-knowledge and experience of the MPC controller system. A data-driven method relies on the fact that abundant process data are available in the DCS database, from which a sample data $\tilde{\mathbf{y}}_{C_{i}}$ is collected for each class $C_{i}$. Then a SVD is carried out on the sample data $\tilde{\mathbf{y}}_{C_{i}}$ to acquire the principal eigenvector, $\phi_{i}$, related to the maximum singular value of the sample data covariance matrix $\operatorname{cov}\left(\tilde{\mathbf{y}}_{C_{i}}\right)$. Each class $C_{i}$ defines a subspace $V_{i}$ spanned by the eigenvectors of $\operatorname{cov}\left(\tilde{\mathbf{y}}_{C_{i}}\right)$, and the class $C_{i}$ or subspace $V_{i}$ is represented by its "feature" eigenvector $\phi_{i}$. This data-driven preprocessing procedure yields the set of the feature eigenvectors $\left\{\boldsymbol{\phi}_{i}\right\}_{i=1}^{g}$ that represent the set of subspaces $\left\{\mathrm{V}_{i}\right\}_{i=1}^{g}$ or classes $\left\{C_{i}\right\}_{i=1}^{g}$.

\subsection{Performance diagnosis using angle-based classifier}

In the performance assessment stage, a SVD is performed on the current monitored data $\tilde{\mathbf{y}}_{\text {II }}$ which lies in its subspace $V_{\text {II }}$ with the feature eigenvector $\phi_{\text {II }}$ corresponding to the maximum singular value of $\operatorname{cov}\left(\tilde{\mathbf{y}}_{\text {II }}\right)$. When a poor performance is detected by the performance assessment procedure, we can examine which of the subspaces $\left\{\mathrm{V}_{i}\right\}_{i=1}^{g}$ is the closest to the subspace $\mathrm{V}_{\mathrm{II}}$ and, therefore, locate the root cause of this poor performance. To do so we define the angle between two subspaces $\mathrm{V}_{\mathrm{u}}$ and $\mathrm{V}_{\mathrm{v}}$. As the eigenvector corresponding to the maximum eigenvalue explains the majority information of a subspace, we use the two feature eigenvectors of the two subspaces to define this angle. Fig. 4 illustrates the two subspaces $\mathrm{V}_{\mathrm{u}}$ and $\mathrm{V}_{\mathrm{v}}$ as well as their two feature eigenvectors $\phi_{\mathrm{u}}$ and $\phi_{\mathrm{v}}$. The angle $\theta_{\mathrm{u}, \mathrm{v}}$ between $\boldsymbol{\phi}_{\mathrm{u}}$ and $\boldsymbol{\phi}_{\mathrm{v}}$ is defined by

$\theta_{\mathrm{u}, \mathrm{v}}=\cos ^{-1}\left(\frac{\boldsymbol{\phi}_{\mathrm{u}}^{T} \boldsymbol{\phi}_{\mathrm{v}}}{\left\|\phi_{\mathrm{u}}\right\| \cdot\left\|\phi_{\mathrm{v}}\right\|}\right)$,

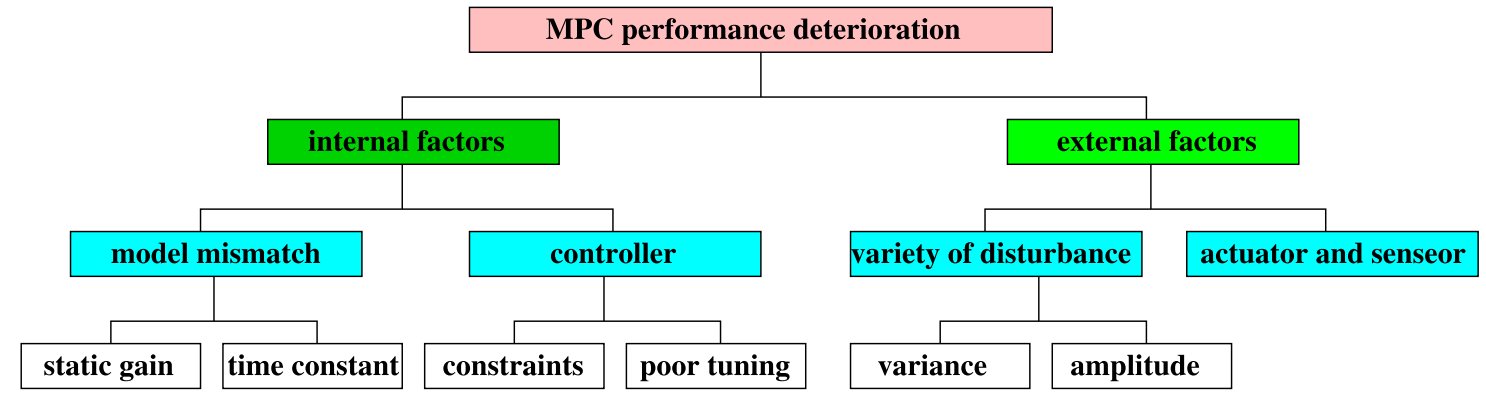

Fig. 3. Illustrative schematic of performance deterioration causes. 


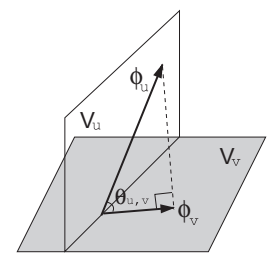

Fig. 4. Illustration of subspace eigenvector angle.

where $\|\bullet\|$ denotes the usual vector norm. The eigenvector angle $\theta_{\mathrm{u}, \mathrm{v}}$ lies in the range of $0-90^{\circ}$. When $\theta_{\mathrm{u}, \mathrm{v}}=0^{\circ}$, the subspace $\mathrm{V}_{\mathrm{u}}$ belongs to the subspace $V_{v}$, i.e. $V_{u} \subset V_{v}$, while $\theta_{u, v}=90^{\circ}$ indicates that $V_{u}$ is orthogonal to $V_{v}$, namely $V_{u} \perp V_{v}$.

We can now summarise the proposed performance diagnosis procedure based on the eigenvector-angle classifier.

(1) A data-driven preprocessing process establishes the set of causes $\left\{C_{i}, \mathrm{~V}_{i}, \phi_{i}\right\}_{i=1}^{g}$, where $C_{i}$ denotes the $i$ th cause or class, $\mathrm{V}_{i}$ is the subspace representing $C_{i}$, and $\phi_{i}$ the feature eigenvector of $\mathrm{V}_{i}$.

(2) During performance assessment of the period $\tilde{\mathbf{y}}_{\mathrm{II}}$, the maximum singular value of $\operatorname{cov}\left(\tilde{\mathbf{y}}_{\mathrm{II}}\right)$ is obtained to assess the current performance, and the related feature eigenvector $\phi_{\mathrm{II}}$ is also extracted to characterise the subspace $V_{\text {II }}$ in which the current data lies.

(3) When a poor performance is detected, the eigenvector angles $\theta_{\mathrm{II}, i}$ between the subspace $\mathrm{V}_{\mathrm{II}}$ and the subspaces $\mathrm{V}_{i}$, for $1 \leq i \leq g$, are calculated. Define

$i^{*}=\arg \min _{1 \leq i \leq g} \theta_{\mathrm{II}, i}$

Then the subspace $V_{i^{*}}$ is the most similar to the subspace $V_{\text {II }}$ and, therefore, the current poor performance is most likely due to the cause $C_{i^{*}}$.

In a statistical decision process, type I error (false positive) and type II error (false negative) are usually used to describe possible errors. Since the above performance diagnosis procedure is entered after a poor performance has been detected and the task is to determine which class that the monitored data set belongs to, we will only consider the misclassification of a class as another. Specifically, define $P\left(C_{i}\right)$ as the accuracy measure for class $C_{i}$, which indicates the probability of the data sets classified as class $C_{i}$ that actually belong to $C_{i}$. Then the false alarm rate (FAR) for class $C_{i}$, which measures the probability of the data sets classified as other classes that actually belong to class $C_{i}$, is defined as

$\operatorname{FAR}\left(C_{i}\right)=1-P\left(C_{i}\right)$

\section{A case study}

We demonstrate the effectiveness of our proposed MPC performance monitoring framework by applying it to the case study involving the Wood-Berry distillation system.

\subsection{Wood-Berry distillation model}

The continuous-time Wood-Berry distillation model is a wellknown two-input two-output transfer function model of a pilotplant distillation column for a methanol-water mixture [22], and it has been used in many previous investigations for process control, monitoring, and identification. This distillation column model is given by

$$
\left[\begin{array}{l}
X_{d}(s) \\
X_{b}(s)
\end{array}\right]=\left[\begin{array}{cc}
\frac{12.8 e^{-s}}{16.7 s+1} & \frac{-18.9 e^{-3 s}}{16.7 s+1} \\
\frac{6.6 e^{-7 s}}{10.9 s+1} & \frac{-19.4 e^{-3 s}}{14.4 s+1}
\end{array}\right]\left[\begin{array}{l}
R(s) \\
S(s)
\end{array}\right]+\left[\begin{array}{c}
\frac{3.8 e^{-8 s}}{10.9 s+1} \\
\frac{4.9 e^{-3 s}}{13.2 s+1}
\end{array}\right] F(s)
$$

In this model, the two output variables are the distillate and bottom compositions, $X_{d}$ and $X_{b}$ [wt\%methanol], respectively, and they are controlled by manipulating the reflux and steam flow rates, $R$ and $S[\mathrm{lb} / \mathrm{min}]$. The feed flow rate, $F$, is an unmeasured disturbance variable.

The closed-loop control simulation was performed using a constrained MPC strategy. The manipulated variables were $u_{1}=R$ and $u_{2}=S$, and the controlled variables were $y_{1}=X_{d}$ and $y_{2}=X_{b}$. Constraints or saturation limits were imposed on the inputs, outputs and input increment velocities. The benchmark parameters of the MPC controller was tuned by trial-and-error to yield a reasonably satisfactory control performance. In particular, in generating the benchmark data, the constraints for the controlled variables, the manipulated variables and the input increments were so set such that they were not active. The model time horizon was set to be 80 . The error weighting and move suppression matrices, $\mathbf{Q}$ and $\mathbf{R}$ in (2), were chosen to be the identity matrix. The unmeasured disturbance $F$ with a variance of 0.01 was added in the simulation but no model-mismatch was applied in generating the benchmark data. The set points of the controlled variables, $X_{d}$ and $X_{b}$, were set to 1 and 0.8 , respectively. The prediction horizon and the control horizon were tuned to be 10 and 1, respectively. Each simulation window was set to 1000 min, which allowed the process reaching the steady state. The sampling period was set to 1 min. The monitored variable set of (6) was established as $\tilde{\mathbf{y}}=\left[\begin{array}{llllll}R & S & e p_{1} & e p_{2} & X_{d} & X_{b}\end{array}\right]^{T}$.

\subsection{Benchmark and training data for the known causes}

Before the data-driven performance assessment could be applied to monitor the MPC control system, one period of the reference data based on the well-tuned controller mentioned in the previous subsection was acquired and used as the benchmark. Furthermore, four prior-known causes to the performance deterioration of the Wood-Berry distillation control system were established, which are described in Table 1 . These four causes of the controller performance deterioration were the variance perturbation of the unmeasured disturbance $\left(C_{1}\right)$, the controller constraint saturation $\left(C_{2}\right)$, the time-varying parameter of the process model $\left(C_{3}\right)$, and the badly tuned parameter of the MPC controller $\left(C_{4}\right)$. Moreover, four training data sets were acquired, one for each class of performance deterioration. The simulated closedloop data of the monitored variable set from the constrained MPC control system corresponding to the benchmark period and the four training periods are shown in Fig. 5, each period containing 1000 data samples. For the $C_{1}$ training period, the variance of the disturbance was 0.015 , compared to the disturbance variance of

Table 1

Classes and their parameters of performance deterioration factors.

\begin{tabular}{|c|c|c|c|}
\hline Class & Operation condition & $\begin{array}{l}\text { Relative parameter/ } \\
\text { variable }\end{array}$ & $\begin{array}{l}\text { Value/ } \\
\text { range }\end{array}$ \\
\hline$C_{1}$ & Disturbance perturbation & Variance & 0.015 \\
\hline$C_{2}$ & $\begin{array}{l}\text { Controller constraint } \\
\text { saturation }\end{array}$ & Output constraint & \pm 0.7 \\
\hline$C_{3}$ & Model-process mismatch & First static gain & 25.6 \\
\hline$C_{4}$ & Bad controller tuning & Control horizon & 3.0 \\
\hline
\end{tabular}



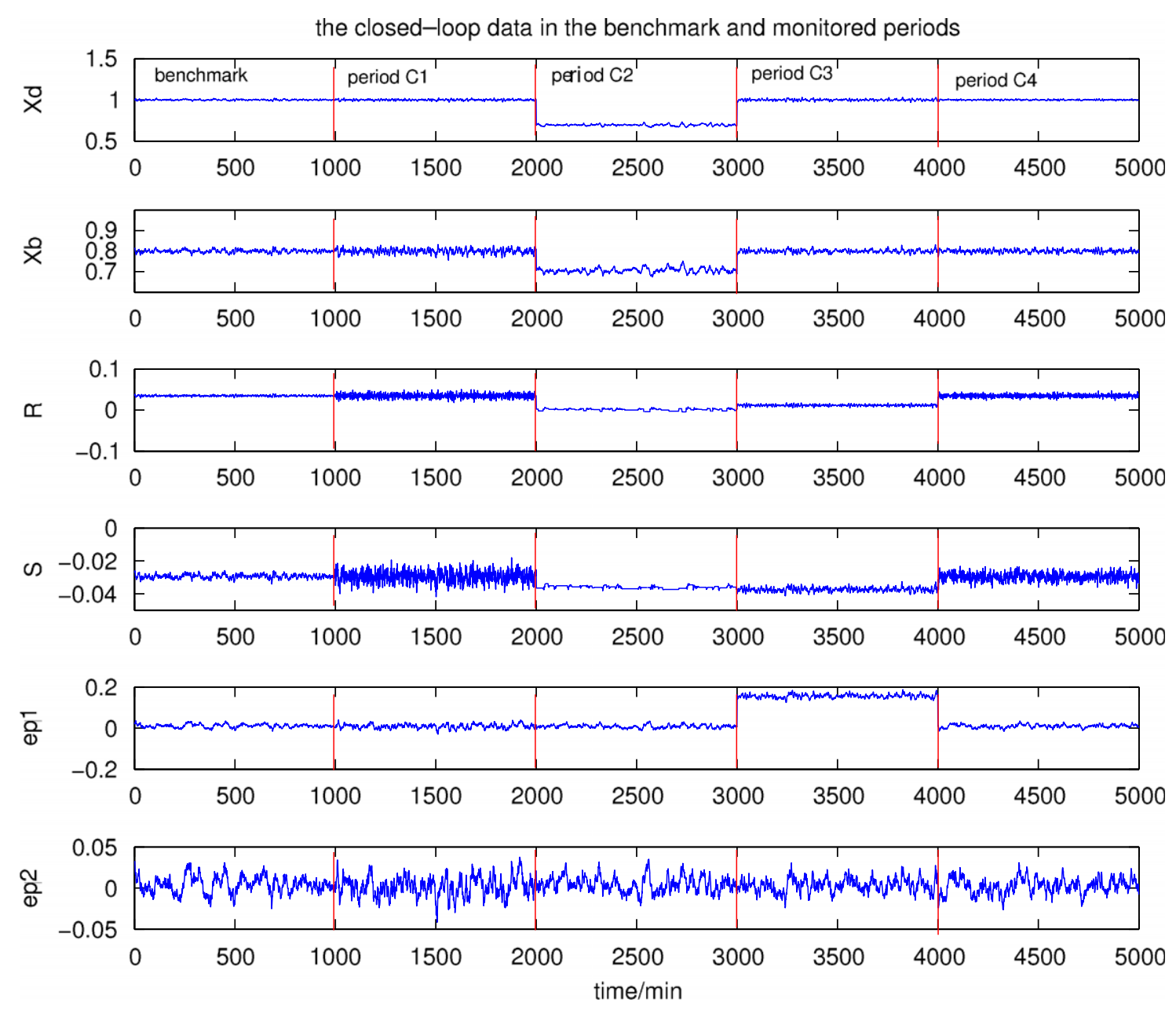

Fig. 5. The closed-loop data in the benchmark and four training periods.

0.01 in the benchmark period. For the $C_{2}$ training period, the controlled variable constraints were set to \pm 0.7 , the manipulated variable limits were set to \pm 0.2 and the constraints on the input increments were \pm 0.05 . This caused the output saturation as the set points, 1.0 and 0.8 , respectively, for the two controlled variables were unreachable. The first gain of the transfer function model (13) in the $C_{3}$ period was changed to be 25.6 which was twice of the nominal value 12.8 , while the control horizon of the $C_{4}$ period was set to 3 in comparison with the control horizon of 1 in the benchmark period.

We first tested the detection power of the proposed 2-norm based performance index (9) on the four training data sets. By performing the SVD on the covariance matrices of the four training periods, the singular values (SVs) for each of the four training periods were extracted, and they are displayed in Fig. 6 in comparison with the SVs of the benchmark period. The performance assessment was then carried out using the performance index $\eta_{\mathrm{m} 2 \mathrm{n}}$ for each period $C_{i}, 1 \leq i \leq 4$. From Fig. 6 , it can be seen that the maximum SV of the benchmark data was 3.08 while the maximum SV of the $C_{1}$ period data was 10.3183 . Thus, the 2 -norm based covariance performance index for the $C_{1}$ period was 0.2985 . Similarly, the maximum SV of the period $C_{2}$ was 4.2340 and the value of $\eta_{\mathrm{m} 2 \mathrm{n}}$ for this period was calculated to be 0.7274 . In the same way, the 2-norm performance index for the closed-loop data of the $C_{3}$ period was found to be 0.7188 , while $\eta_{\mathrm{m} 2 \mathrm{n}}=0.6718$ was obtained for the $C_{4}$ period data. The values of $\eta_{\mathrm{m} 2 \mathrm{n}}$ for the four training periods are listed in Table 2 , which demonstrate that the deteriorated performance of the corresponding process control monitored were correctly detected by the proposed 2-norm based covariance performance assessment method. The feature eigenvectors $\left\{\phi_{i}\right\}_{i=1}^{4}$ corresponding to the four maximum SVs of the four training classes $\left\{C_{i}\right\}_{i=1}^{4}$ were also extracted, ready for the use in performance diagnosis.

We also carried out the MPC controller performance assessment on the same data of the four training periods using the historical performance benchmark [18] and the determinant based covariance benchmark [20]. The history performance index method [18] specifically focuses on the MPC performance assessment using a quadratic objective function of the controller's inputs and outputs. The values of the history performance index obtained for assessing the four training periods are also given in Table 2 . The results of the performance assessment using the determinant based benchmark [20], $\eta_{\mathrm{det}}$ of (1), are also shown in Table 2 in comparison with the other two performance assessment methods. From Table 2, it can be seen that the $\eta_{\text {det }}$ and $\eta_{\mathrm{m} 2 \mathrm{n}}$ based performance assessment methods performed satisfactorily and they all correctly detected the deterioration of the controller performance. It could argue that the determinant based covariance performance index $\eta_{\mathrm{det}}$ is more sensitive to the performance deviation from the benchmark than the 2-norm based covariance performance index $\eta_{\mathrm{m} 2 \mathrm{n}}$, as the former counts the energy of all the eigenvalues while the latter only uses the energy of the largest eigenvalue. However, our proposed performance assessment method forms an integrated part of the performance monitoring procedure and offers a significant advantage in performance diagnosis. For the $C_{4}$ class of training data, the history performance index yielded a value of 
a

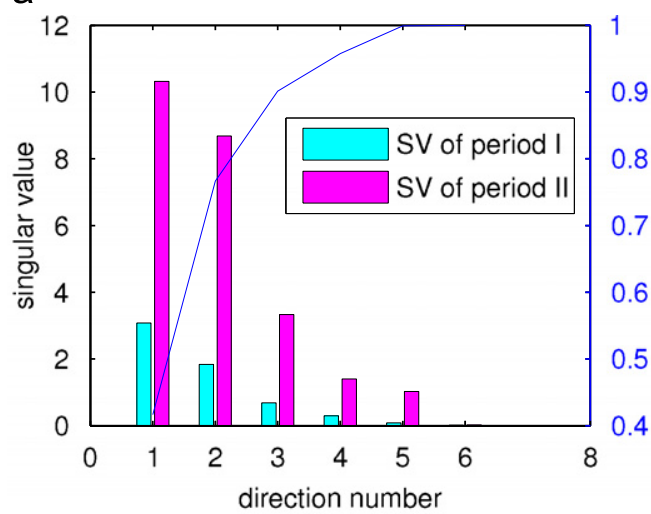

C

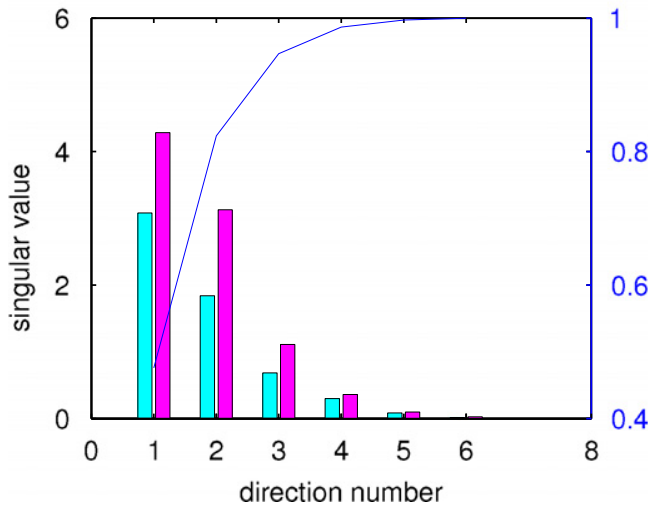

b

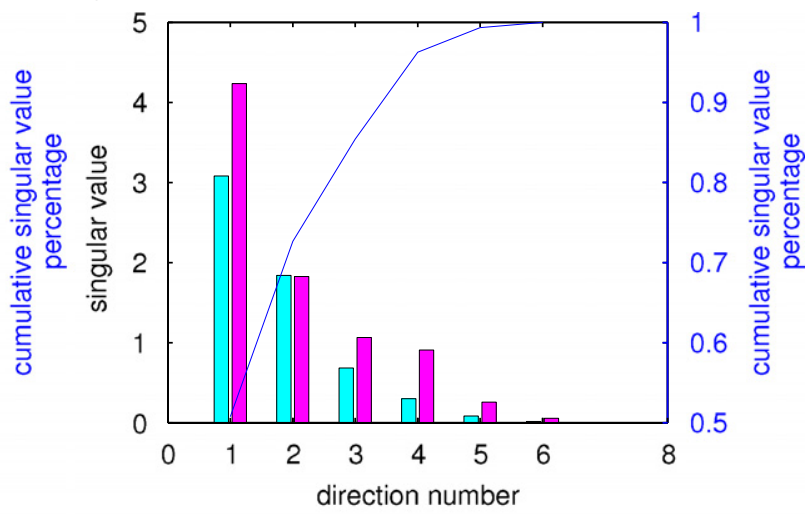

d

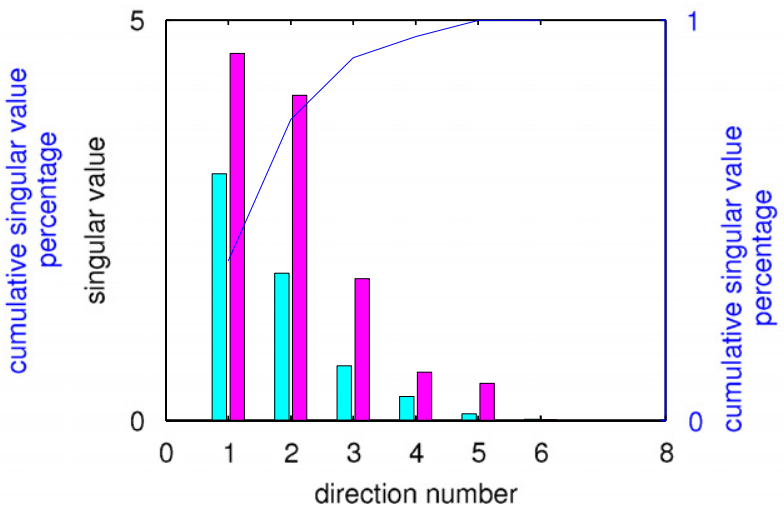

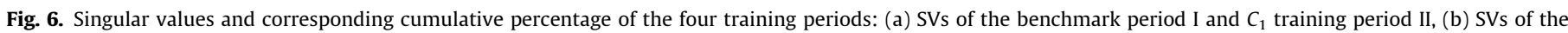

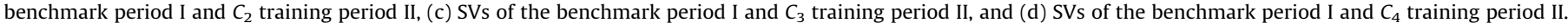

Table 2

Performance comparison of three performance assessment methods.

\begin{tabular}{lllll}
\hline Monitored period & $C_{1}$ & $C_{2}$ & $C_{3}$ & $C_{4}$ \\
\hline Proposed 2-norm based covariance index & 0.2985 & 0.7274 & 0.7188 & 0.6718 \\
History performance index index [18] & 0.4319 & 0.0011 & 0.5928 & 0.9497 \\
Determinant based covariance index [20] & 0.0071 & 0.0173 & 0.1233 & 0.0213 \\
\hline
\end{tabular}

0.9497, which may not be considered a clear detection of performance deterioration since the performance index could be regarded as too close to the unity.

\subsection{Experimental results}

In Subsection 4.2, the benchmark data were acquired and the database for the four classes of performance deterioration were established, which were denoted as $\left\{C_{i}, V_{i}, \phi_{i}\right\}_{i=1}^{4}$ with $V_{i}$ the subspace representing the class $C_{i}$ and $\phi_{i}$ the feature eigenvector of $\mathrm{V}_{i}$. To test our performance monitoring procedure and, in particular, to validate the effectiveness of our eigenvector-angle based classifier for performance diagnosis, four groups of the additional data were generated to represent different operation conditions, and they were labeled as FP1, FP2, FP3 and FP4, respectively. All the data were divided into periods with each period containing 1000 data samples. The group FP1 data contained the five periods, which were obtained by setting the disturbance variance to $0.012,0.013,0.014,0.015$ and 0.018 , respectively. The four periods of the group FP2 data were generated by setting the constraints of the controlled variables to $\pm 0.7, \pm 0.6, \pm 0.5$ and \pm 0.4 , respectively, while the constraints on the inputs and input increments were unchanged as those specified for the $C_{2}$ training period. The five periods of the group FP3 data were obtained with the first gain of the transfer function model (13) increased by $60 \%$, $80 \%, 90 \%, 100 \%$ and $120 \%$, respectively, over the nominal value given in (13). Setting the control horizon to 2 and 3, respectively, yielded the two periods of the group FP4 data.

The proposed performance assessment method was applied to all the four groups of data, and the corresponding values of the 2-norm based covariance performance index are plotted in Fig. 7, where the first point at the period 0 denotes the performance index of the benchmark period. As can be seen from Fig. 7, the proposed performance assessment method correctly detected the performance deterioration in each period of the monitored data in every group. As expected, increase in the disturbance variance worsened the performance, as can be seen clearly from the plot for the five periods of the group FP1 data in Fig. 7. When the disturbance variance increased to 0.018 , the value of the performance index $\eta_{\mathrm{m} 2 \mathrm{n}}$ decreased to 0.0161 . Similarly, the other three plots in Fig. 7 clearly demonstrate the serious performance degradation caused by controller saturation, model-process mismatch and bad controller tuning, respectively. The values of $\eta_{\mathrm{det}}$ obtained for all the four groups of data, not shown here, also confirm that the determinant based covariance index [20] correctly detected the performance deterioration in each period of the monitored data in every group. ${ }^{1}$

As the performance assessment detected a poor performance in each period of the monitored data in every group, the performance

\footnotetext{
${ }^{1}$ In fact, as shown previously, the determinant based covariance performance index $\eta_{\text {det }}$ is more sensitive to the performance deviation from the benchmark than the 2-norm based covariance performance index $\eta_{\mathrm{m} 2 \mathrm{n}}$. Our proposed performance assessment method, however, forms an integrated part of the performance monitoring procedure and can directly aid performance diagnosis.
} 

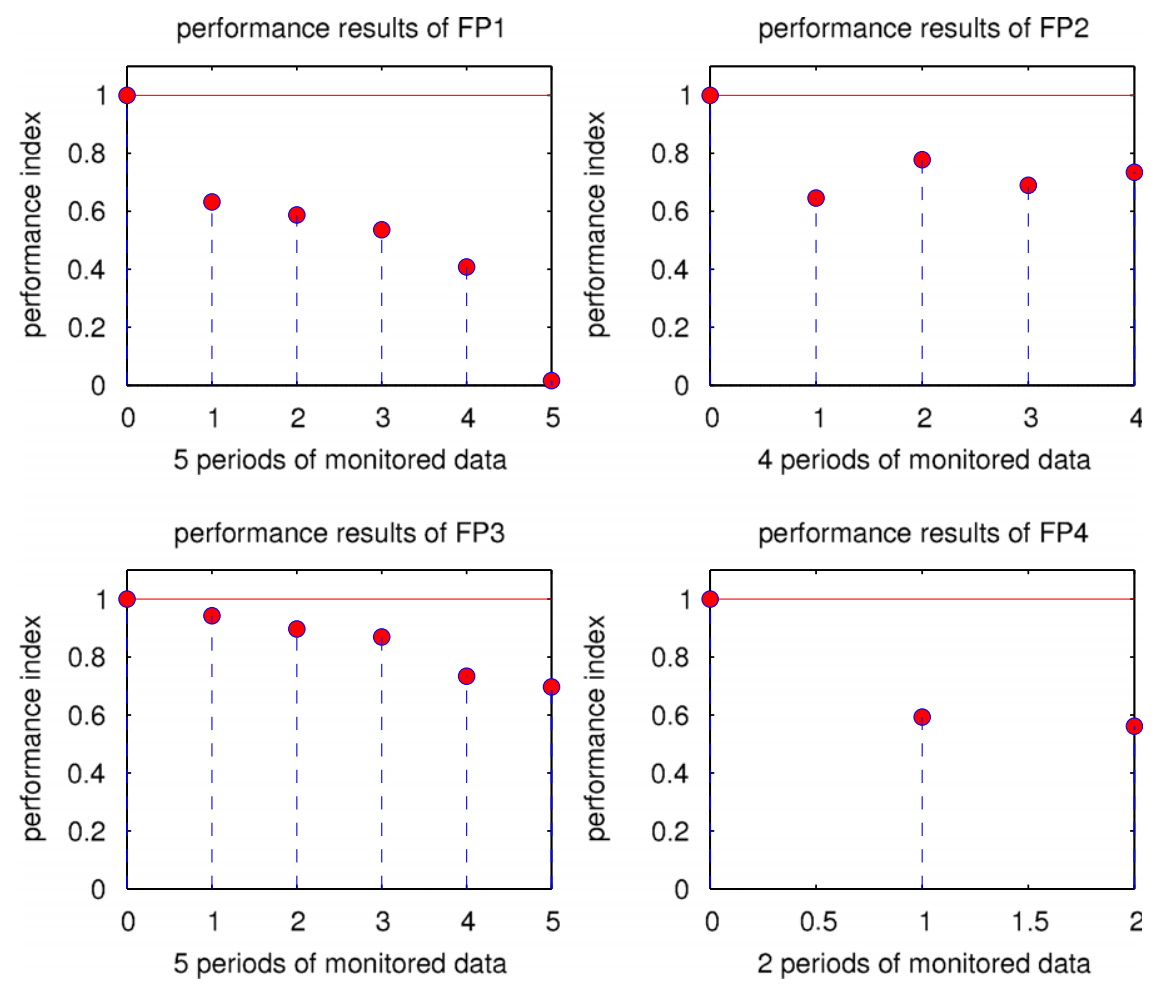

Fig. 7. Performance assessment results for the four groups of the monitored data using the proposed 2-norm based covariance index.
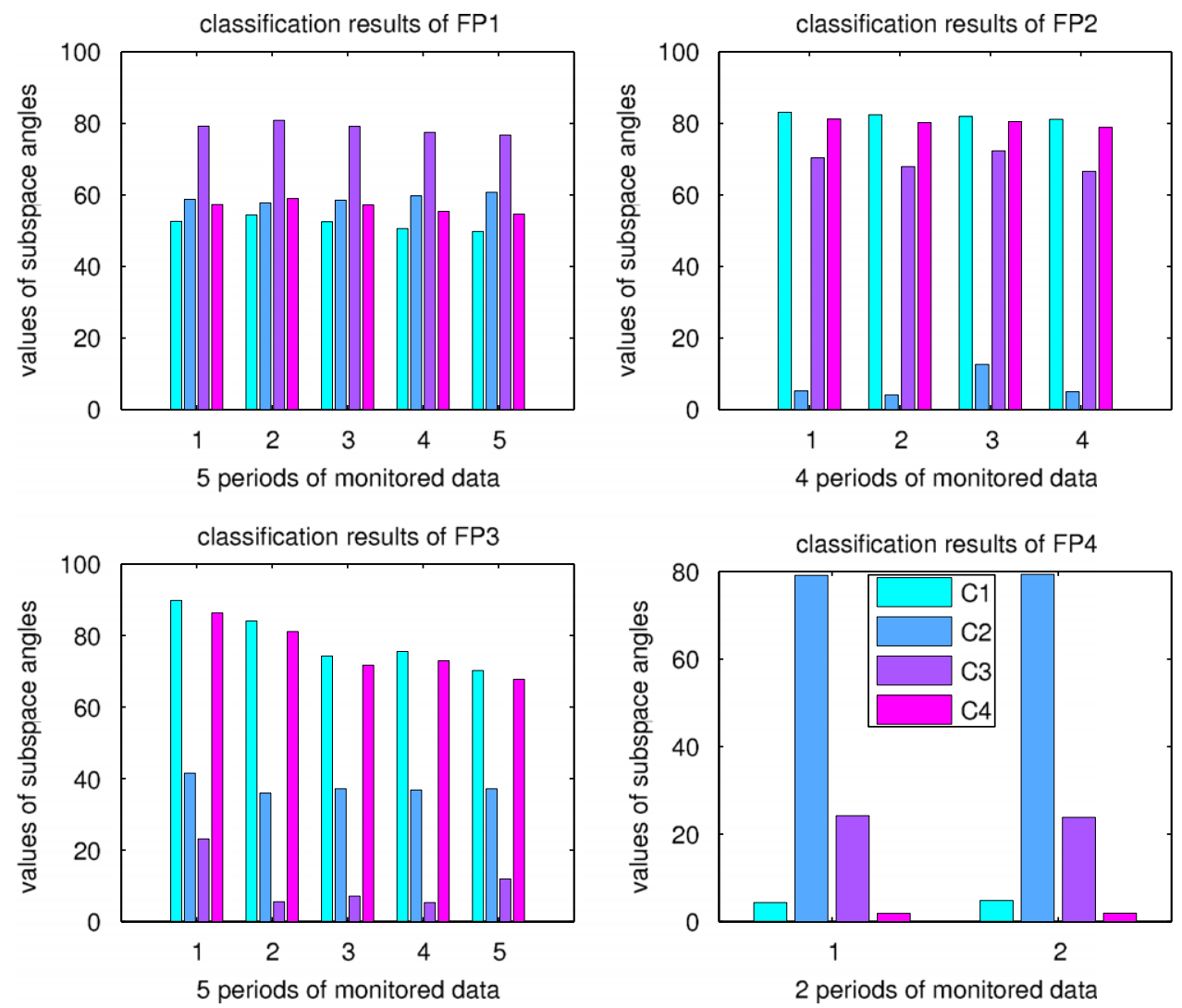

Fig. 8. Performance diagnosis results for the four groups of the monitored data using the proposed eigenvector-angle based classifier.

diagnosis procedure was entered to locate the root cause of the poor performance for each period of the monitored data. The eigenvector angles between the feature eigenvector for each period of the monitored data in every group and the four pre-prepared feature eigenvectors $\left\{\phi_{i}\right\}_{i=1}^{4}$ are depicted in Fig. 8. The results of Fig. 8 demonstrate that the proposed performance diagnosis 
Table 3

Classification results over 1600 periods of the monitored data using the proposed eigenvector-angle based classifier.

\begin{tabular}{|c|c|c|c|c|c|}
\hline \multicolumn{2}{|c|}{ Monitored data sets } & \multicolumn{4}{|c|}{ Classified as (\%) } \\
\hline & & $C_{1}$ & $C_{2}$ & $C_{3}$ & $C_{4}$ \\
\hline \multicolumn{2}{|l|}{ FP1 (500) } & 75.4 & 0 & 24.6 & 0 \\
\hline \multicolumn{2}{|l|}{ FP2 (500) } & 0 & 100 & 0 & 0 \\
\hline \multicolumn{2}{|l|}{ FP3 (500) } & 0 & 0 & 100 & 0 \\
\hline \multicolumn{2}{|l|}{ FP4 (100) } & 0 & 0 & 0 & 100 \\
\hline Accuracy & $P\left(C_{i}\right)$ & 0.754 & 1 & 1 & 1 \\
\hline FAR & $\operatorname{FAR}\left(C_{i}\right)$ & 0.246 & 0 & 0 & 0 \\
\hline
\end{tabular}

method successfully found the root causes of performance degradation for all the four groups of data. Specifically, the eigenvector angle plot for the group FP1 shown in Fig. 8 indicated that the poor performance for all the five periods of the group FP1 data were caused by the external disturbance, i.e. due to the $C_{1}$ cause. Similarly, the eigenvector angle plot for all the four periods of the group FP2 data depicted in Fig. 8 revealed that the cause for poor performance was the $C_{2}$ controller saturation. In the same way, the causes for the poor performance in the groups FP3 and FP4 were located to be the $C_{3}$ model mismatch and $C_{4}$ inappropriate tuning of the controller, respectively.

To gain some statistical accuracy measure of the proposed performance monitoring procedure, large number of data sets was generated with each data period to be monitored containing again 1000 data samples. Specifically, 500 periods of the group FP1 data were produced by randomly choosing the disturbance variance from $[0.012,0.037]$, while 500 periods of the group FP2 data were derived by setting the constraint magnitudes for the controlled variables randomly in $[0.35,0.8]$. By randomly increasing the first gain of the transfer function (13) in the range of $20-300 \%$ over the nominal value, 500 periods of the group FP3 data were generated. Similarly, choosing the control horizon randomly 100 times in the range of 2-9 produced 100 periods of the group FP4 data. Each of the 1600 periods of the monitored data was assessed and the 2-norm based covariance performance index correctly detected performance deterioration in all the 1600 periods of the monitored data. The performance diagnosis was then carried out using the proposed eigenvector-angle based classifier for all the 1600 periods of the monitored data, and the classification results obtained are summarised in Table 3, where it can be seen that $75.4 \%$ of the group FP1 data sets were correctly classified as the $C_{1}$ class while $24.6 \%$ of the group FP1 data sets were mistakenly classified as the $C_{3}$ class, ${ }^{2}$ yielding the FAR for $C_{1}$ class $\operatorname{FAR}\left(C_{1}\right)=0.246$. However, the other three groups of the data sets were correctly classified into the corresponding classes completely. Thus, the overall classification accuracy is

overall accuracy $=(0.754 \times 500+500+500+100) / 1600=92.31 \%$, which guarantees the overall FAR to be $1-0.9231=7.69 \%$.

\section{Conclusions}

A data-based MIMO performance monitoring approach for model predictive controllers has been proposed. Model predictive errors have been included into the monitored variable set to increase the sensitivity of the performance index to the changes

\footnotetext{
${ }^{2}$ The reasons for small number of the $\mathrm{C} 1$ data sets been classified as the $\mathrm{C} 3$ class are highly complex. We could only suggest that the effects of the variance perturbation in the unmeasured disturbance $\left(C_{1}\right)$ in these data might be similar to the effects of the parameter variation in the process model $\left(C_{3}\right)$.
}

in the process model, constraint saturation and disturbance. A 2-norm based covariance benchmark has been proposed for evaluating the performance of the MPC controller monitored. The proposed performance assessment method uses SVD to extract the major information of the data covariance matrix as well as the principal eigenvector corresponding to the maximum singular value. A novel performance diagnosis method has been proposed based on the principal eigenvector-angle classifier to locate the root cause of poor performance detected. This diagnosis method relies on a user-predefined database establishing the set of the class principal eigenvectors that defines the causes of poor performance, based on priori knowledge and experience of the process. The proposed approach has been applied to a case study of the WoodBerry distillation column process. The effectiveness of both the performance assessment and diagnosis procedures has been validated.

The proposed method represents a systematic approach for controller performance monitoring in which both the performance assessment and diagnosis procedures are naturally integrated. The performance assessment provides the operator not only a quantitative indicator whether or not the controller works as expected but also further diagnosis information. Like other data-driven methods, the proposed approach is flexible in MPC performance monitoring since, if the performance of the controller monitored turns out to be better than the benchmark of a golden operational data, the benchmark can be replaced. Many critical issues of MPC controller performance monitoring, however, warrant further investigation. These include determining the confidence limit which is the watershed of the performance deterioration, and indepth classification of the causes for poor performance as well as extending to nonlinear MPC controller performance monitoring.

\section{References}

[1] T.J. Harris, Assessment of closed-loop performance, Canadian Journal of Chemical Engineering 67 (10) (1989) 856-861.

[2] T.J. Harris, F. Boudreau, J.F. Macgregor, Performance assessment of multivariate feedback controllers, Automatica 32 (11) (1996) 1505-1518.

[3] T.J. Harris, C.T. Seppala, L.D. Desborough, A review of performance monitoring and assessment techniques for univariate and multivariate control systems, Journal of Process Control 9 (1) (1999) 1-17.

[4] B.-S. Ko, T.F. Edgar, Performance assessment of multivariable feedback control systems, Automatica 37 (6) (2001) 899-905.

[5] T.J. Harris, C.T. Seppala, Recent developments in controller performance monitoring and assessment techniques, in: AICHE Symposium Series, Issue 326, 2002, pp. 208-222.

[6] M. Jelali, An overview of control performance assessment technology and industrial applications, Control Engineering Practice 14 (5) (2006) 441-466.

[7] S.J. Qin, J. Yu, Recent developments in multivariable controller performance monitoring, Journal of Process Control 17 (3) (2007) 221-227.

[8] L. Desborough, R. Miller, Increasing customer value of industrial control performance monitoring-Honeywells experience, in: AICHE Symposium Series, Issue 326, 2002, pp. 169-189.

[9] J. Gao, R. Patwardhan, K. Akamatsu, Y. Hashimoto, G. Emoto, S.L. Shah, B. Huang, Performance evaluation of two industrial MPC controllers, Control Engineering Practice 11 (12) (2003) 1371-1387.

[10] S.J. Qin, T.A. Badgwell, A survey of industrial model predictive control technology, Control Engineering Practice 11 (7) (2003) 733-764.

[11] S.L. Shah, R. Patwardhan, B. Huang, Multivariate controller performance analysis: methods, applications and challenges, in: AICHE Symposium Series, Issue 326, 2002, pp. 190-207.

[12] B. Huang, Multivariate statistical methods for control loop performance assessment, Ph.D. Thesis, Department of Chemical Engineering, University of Alberta, Canada, 1997.

[13] C. Zhao, Y. Zhao, H. Su, B. Huang, Economic performance assessment of advanced process control with LQG benchmarking, Journal of Process Control 19 (4) (2009) 557-569.

[14] R.S. Patwardhan, S.L. Shah, Issues in performance diagnostics of model-based controllers, Journal of Process Control 12 (3) (2002) 413-427.

[15] Q. Zhang, S. Li, Performance monitoring and diagnosis of multivariable model predictive control using statistical analysis, Chinese Journal of Chemical Engineering 14 (2) (2006) 207-215.

[16] A. AlGhazzawi, B. Lennox, Model predictive control monitoring using multivariate statistics, Journal of Process Control 19 (2) (2009) 314-327. 
[17] R.S. Patwardhan, S.L. Shah, K.Z. Qi, Assessing the performance of model predictive controllers, Canadian Journal of Chemical Engineering 80 (5) (2002) 954-966.

[18] J. Schäfer, A. Cinar, Multivariable MPC system performance assessment, monitoring, and diagnosis, Journal of Process Control 14 (2) (2004) 113-129.

[19] C.A. McNabb, S.J. Qin, Projection based MIMO control performance monitoring: I-covariance monitoring in state space, Journal of Process Control 13 (8) (2003) 739-757.

[20] J. Yu, S.J. Qin, Statistical MIMO controller performance monitoring, part I: datadriven covariance benchmark, Journal of Process Control 18 (3-4) (2008) 277-296.

[21] J. Yu, S.J. Qin, Statistical MIMO controller performance monitoring, part II: performance diagnosis, Journal of Process Control 18 (3-4) (2008) 297-319.

[22] F. Loquasto, D.E. Seborg, Monitoring model predictive control systems using pattern classification and neural networks, Industrial \& Engineering Chemistry Research 42 (20) (2003) 4689-4701.

[23] F. Loquasto, D.E. Seborg, Model predictive controller monitoring based on pattern classification and PCA, in: Proceedings of the 2003 American Control Conference, vol. 3, Denver, Colorado, June 4-6, 2003, pp. 1968-1973.

[24] O.A.Z. Sotomayor, D. Odloak, Performance assessment of model predictive control systems, in: Proceedings of the 2006 International Symposium on Advanced Control of Chemical Processes, vol. 6, Part 1, Brazil, April 2-5, 2006 pp. 875-880.

[25] N.F. Thornhill, A. Horch, Advances and new directions in plant-wide controller performance assessment, in: Proceedings of the 2006 International Symposium on Advanced Control of Chemical Processes, vol. 6, Part 1, Brazil, April 25, 2006, pp. 29-36.

[26] J.R. Webber, Y.P. Gupta, A closed-loop cross-correlation method for detecting model mismatch in MIMO model-based controllers, ISA Transactions 47 (4) (2008) 395-400.

[27] Y. Zhao, Y. Gu, H. Su, B. Huang, Extended prediction error approach for MPC performance monitoring and industrial applications, in: Proceedings of the 17th IFAC World Congress, vol. 17, Part 1, Korea, July 6-11, 2008, pp. $14894-$ 14899.

[28] M.L. Tyler, M. Morari, Performance monitoring of control systems using likelihood methods, in: Proceedings of the 1995 American Control Conference, vol. 2, Seattle, Washington, June 21-23, 1995, pp. 1245-1249.

[29] R.H. Julien, M.W. Foley, W.R. Cluett, Performance assessment using a model predictive control benchmark, Journal of Process Control 14 (4) (2004) 441-456.

[30] B. Huang, Bayesian methods for control loop monitoring and diagnosis, Journal of Process Control 18 (9) (2008) 829-838.

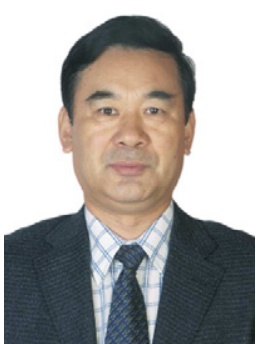

Xuemin Tian received his Bachelor of Engineering from Huadong Petroleum Institute, Dongying, China, in January 1982, and his M.S. degree from Beijing University of Petroleum, Beijing, China, in June 1994. From September 2001 to June 2002, he served as visiting professor at Central of Process Control, University of California in Santa Barbara. He is a Professor of Process Control at China University of Petroleum (Hua Dong).

Professor Tian's research interests are in modelling, advanced process control and optimisation for petrolchemical processes as well as fault detection and diagnosis, and process monitoring.

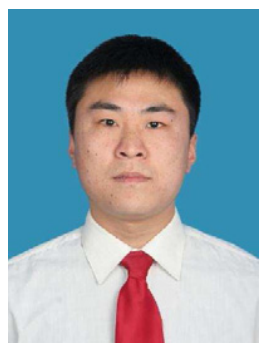

Gongquan Chen was born in Shandong, China, on September 28, 1983. He received his B.Sc. degree in 2006 and his M.S. degree in July 2009, both from China University of Petroleum.

Since 2009, he serves as an Assistant Engineer in China MCC20 Group Corp., Ltd. His current research interests focus on controller performance assessment and diagnosis in industrial processes.

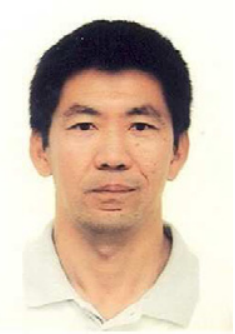

Sheng Chen received his BEng degree from Huadong Petroleum Institute, Dongying, China, in January 1982, and his PhD degree from the City University, London, UK, in September 1986, both in Control Engineering. He was awarded the Doctor of Sciences (DSc) degree by the University of Southampton, Southampton, UK, in 2004

From October 1986 to August 1999, he held research and academic appointments at the University of Sheffield, the University of Edinburgh and the University of Portsmouth, all in UK. Since September 1999, he has been with the School of Electronics and Computer Science, the University of Southampton, UK, where he holds the post of Professor of Intelligent Systems and Signal Processing. Professor Chen's research interests include wireless communications, adaptive signal processing for communications, machine learning, evolutionary computation methods, and intelligent control systems. He has published over 400 research papers.

Dr Chen is a Fellow of IET and a Fellow of IEEE. In the database of the world's most highly cited researchers, compiled by Institute for Scientific Information (ISI) of the USA, Dr. Chen is on the list of the highly cited researchers in the engineering category. 